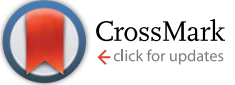

Cite this: RSC Adv., 2017, 7, 17711
Received 5th January 2017 Accepted 12th February 2017

DOI: 10.1039/c7ra00189d

rsc.li/rsc-advances

\section{Poly(L-lysine) modified zein nanofibrous membranes as efficient scaffold for adhesion, proliferation, and differentiation of neural stem cells $\dagger$}

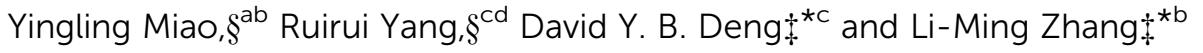

Excellent biocompatibility and bioactivity are necessary requirements for a scaffold for nerve repair and regeneration. Natural plant protein zein was chosen as the primary material and poly(L-lysine), which is composed of common amino acids in the human body, was used to modify it. Poly(L-lysine) modified zein (ZPLL) with different PLL contents of $1.46 \%, 3.57 \%$, and $6.18 \%$ was synthesized and nanofibrous membranes were prepared by electrospinning. The hydrophilicity of the membranes improved with an increase of PLL content. The biodegradability of the membranes was proved by in vitro experiments. Compared with pure zein membranes, ZPLL membranes can efficiently improve cell viability, adhesion, proliferation, and differentiation of neural stem cells (NSCs) and the effect of PLL content was further investigated. The results show that when the PLL content was 3.57\%, cell adhesion and proliferation proved to be the best and most differentiated toward mature neurons with extensive neurite formation and astrocytes rather than oligodendrocytes. The ZPLL was made into nerve conduits for future study and they may be a promising biomaterial for nerve repair and regeneration.

\section{Introduction}

About $2.8 \%$ of patients with trauma suffer from peripheral nerve injury which may result in loss of perceptive and muscle function. ${ }^{1-6}$ In cases of severe injuries causing complete nerve transection, surgical treatment is required. End-to-end suturing usually can solve the problem with small gaps. ${ }^{7}$ However, large gaps longer than $3 \mathrm{~cm}$ require an implant to be bridged, ${ }^{8}$ Autografts are commonly used in clinics despite source limits and may cause damage to the donor. ${ }^{89}$ Therefore, alternative materials capable of matching or exceeding the performance of autografts are attracting wide attention. ${ }^{\mathbf{1 0 , 1 1}}$

\footnotetext{
${ }^{a}$ Department of Polymer and Materials Science, School of Chemistry, Key Laboratory for Polymeric Composite and Functional Materials of Ministry of Education, Guangdong Provincial Key Laboratory for High Performance Polymer-based Composites, Sun Yat-sen University, Guangzhou 510275, China

${ }^{b}$ School of Materials Science and Engineering, Sun Yat-sen University, Guangzhou 510275, China. E-mail: ceszhlm@mail.sysu.edu.cn

${ }^{c}$ Research Center of Translational Medicine, The First Affiliated Hospital, Guangdong Provincial Key Laboratory of Orthopedics and Traumatology, Sun Yat-sen University, Guangzhou 510080, China.E-mail: dengyub@mail.sysu.edu.cn

${ }^{d}$ Department of Pathophysiology, Taishan Medical University, Taian 271016, China $\dagger$ Electronic supplementary information (ESI) available. See DOI: 10.1039/c7ra00189d

$\S$ Both authors contributed equally to this work.

\$ Both corresponding authors contributed equally to this work.
}

Synthetic polymers such as PCL, ${ }^{12,13}$ PEG, ${ }^{14}$ PLLA, ${ }^{15-17}$ PU, ${ }^{18}$ and natural polymers like chitosan, ${ }^{19,20}$ gelatin, ${ }^{21,22}$ silk fibroin, ${ }^{23}$ collagen, ${ }^{24,25}$ etc. have been used to prepare nerve conduits. At the same time, these polymers are often modified with Arg-GlyAsp (RGD) peptide to improve biocompatibility and bioactivity. ${ }^{26}$ Besides, poly(L-lysine) (PLL) was reported to be conducive to the attachment, proliferation, and differentiation of nerve cells on materials at certain density. ${ }^{\mathbf{1 4 , 2 7}}$ Compared with RGD, PLL could be artificially synthesized with lower cost and is easier to be chemically modified, which is promising for nerve repairs.

Zein is a promising material in the biomedical field as it is a natural protein from corn with excellent biocompatibility and biodegradability. ${ }^{28,29}$ However, application is limited by its hydrophobic property since over $50 \%$ of the amino acids composing zein are nonpolar amino acids such as leucine, proline, alanine, and phenylalanine. ${ }^{30}$ Zein was used to blend with other materials such as polysaccharides to overcome its hydrophobic property. ${ }^{31}$ However, since the blended materials were simply combined by physical mixing, phase separation may occur when they are used. ${ }^{32}$ Because of this, many researchers consider chemical modification as a better way to optimize these materials. ${ }^{33}$ However, few reports exist concerning modification of zein by chemical routines.

In this work, we modified zein with PLL, the result of which has a similar structure to protein through a chemical 
modification method, and then electrospun it into nanofibrous membranes. Neural stem cells were used to evaluate biocompatibility of the membranes. Our results indicated that the PLL modified zein nanofibrous membranes can efficiently improve cell viability, adhesion, proliferation, and differentiation of NSCs. The ZPLLs could be prepared into nerve conduits for future study and might be a promising biomaterial for nerve repair and regeneration.

\section{Experimental}

\subsection{Materials and methods}

Zein (grade Z3625, 22-24 kDa) and collagenase type I were purchased from Sigma-Aldrich. $N^{\varepsilon}$-Carbobenzoxy-L-lysine (98\%) and trifluoroacetic acid (TFA, 99\%) were purchased from Aladdin Chemical Company. Triphosgene (99.5\%) was purchased from Xiya Reagent. 1,1,1,3,3,3-Hexafluoro-2-propanol (HFIP, 99\%) and hydrogen bromide (33 wt $\%$ in acetic acid) were purchased from Energy Chemical Company. Tetrahydrofuran (THF, Shanghai Sinopharm Chemical Reagent Corp., 99.5\%) was dried by refluxing over sodium/benzophenone and distilled just prior to use. Dimethylformamide (DMF, Shanghai Sinopharm Chemical Reagent Corp., 99.5\%) was distilled from calcium hydride under reduced pressure and stored over molecular sieves.

NSCs (neural stem cells) were obtained from adult SD rats (Laboratory Animal Center of Sun Yat-sen University, Guangzhou, China), as previously described. ${ }^{34,35}$ All experimental and animal-handling procedures were approved by the First Affiliated Hospital, Sun Yat-sen University Ethics Committee on the Use of Live Animals in Teaching and Research and were conducted in accordance with the Guide to the Care and Use of Experimental Animals by the National Research Council (1996, United States). Growth medium containing DMEM/F12 medium, StemPro neural supplement, epidermal growth factor (EGF), basic fibroblastic growth factor (bFGF), and GlutaMAX were purchased from Life Technologies. Penicillinstreptomycin solution, accutase and BSA were purchased from Sigma. Triton X-100 was purchased from Fluka. 4',6-Diamidino2-phenylindole dilactate (DAPI) was purchased from Invitrogen.

${ }^{1} \mathrm{H}$ NMR spectra were recorded on an INOVA 500 NB superconducting Fourier transform nuclear magnetic resonance spectrometer (Varian, USA). FT-IR spectra were recorded on a Nicolet 670 FT-IR spectrometer (Thermo Nicolet, USA). Elemental analysis was carried out by a Vario EL (Elementar, France) element analyzer. XRD analysis was carried out by an Empyrean X-ray diffractometer (PANalytical, Netherlands). A theta goniometer was used to scan from $3-60^{\circ}$.

The synthetic route of PLL modified zein is shown in Scheme 1.

\subsection{Preparation of $N^{\varepsilon}$-carbobenzoxy-L-lysine $N$ - carboxyanhydride (ZLL-NCA)}

$N^{\varepsilon}$-Carbobenzoxy-L-lysine $(10.00 \mathrm{~g}, 37.55 \mathrm{mmol})$ was suspended in $200 \mathrm{~mL}$ anhydrous THF. Triphosgene $(6.00 \mathrm{~g}, 20.22 \mathrm{mmol})$ was added at $50{ }^{\circ} \mathrm{C}$ under $\mathrm{N}_{2}$ protection. The reaction turned
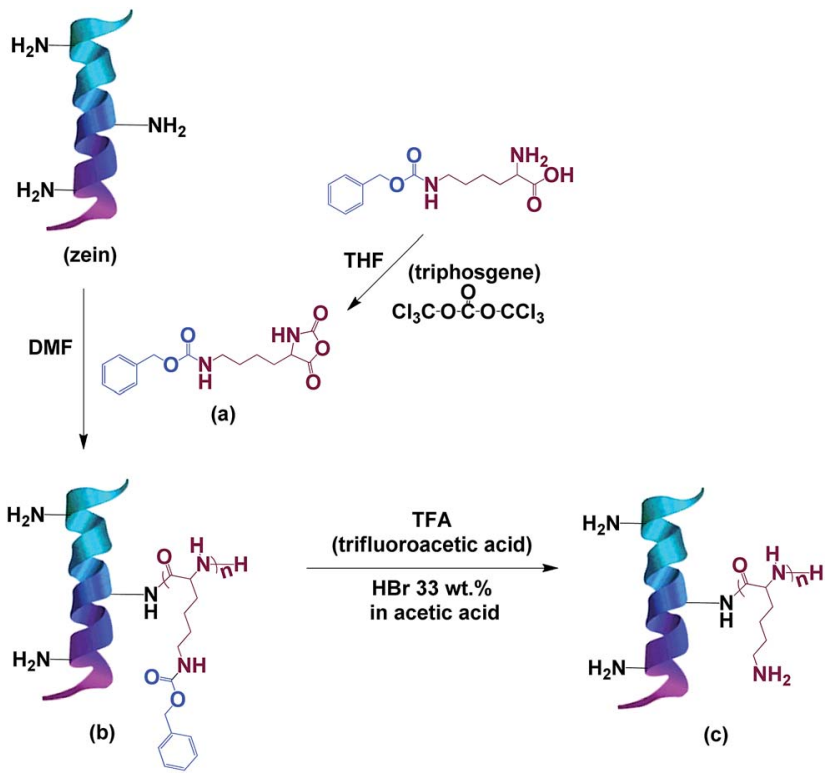

(c)

Scheme 1 Synthesis of poly(L-lysine) modified zein.

clear in a short time and then cooled to room temperature after $30 \mathrm{~min}$. THF was removed by rotary evaporation. A white solid was obtained and dissolved in $200 \mathrm{~mL}$ ethyl acetate. That solution was washed with cold saturated $\mathrm{NaHCO}_{3}$ solution until no more gas formed, followed by washing three times with cold deionized water. The organic layer was dried over $\mathrm{MgSO}_{4}$ and evaporated under vacuum. A white solid was obtained as $N^{\varepsilon}$ carbobenzoxy-L-lysine $N$-carboxyanhydride (ZLL-NCA). Yield: $82 \%$.

\subsection{Preparation of poly $\left(N^{\varepsilon}\right.$-carbobenzoxy-L-lysine $)$ grafted zein (ZPZLL)}

$N^{\varepsilon}$-Carbobenzoxy-L-lysine $N$-carboxyanhydride $(0.50 \mathrm{~g}, 1.71$ mmol) was dissolved in $30 \mathrm{~mL}$ anhydrous DMF. A certain amount of zein was added under $\mathrm{N}_{2}$ protection and the mixture was stirred for 3 days. Diethyl ether was used to precipitate the product and a yellow solid was obtained. Three different samples were synthesized by adding different amounts of zein, as shown in Table 1.

\subsection{Preparation of poly(L-lysine) grafted zein (ZPLL)}

ZPZLL (1.00 g) was dissolved in $10 \mathrm{~mL}$ trifluoroacetic acid (TFA). Hydrogen bromide, $33 \mathrm{wt} \%$ in acetic acid $(3.0 \mathrm{~mL})$, was add dropwise at $0{ }^{\circ} \mathrm{C}$. After reacting for $2 \mathrm{~h}$, the mixture was condensed under vacuum to remove the solvents. The solid

Table 1 Synthesis conditions of ZPZLLS

\begin{tabular}{lllll}
\hline Sample & ZLL-NCA $(\mathrm{g})$ & Zein $(\mathrm{g})$ & DMF $(\mathrm{mL})$ & Yield\% \\
\hline ZPZLL-1 & 0.50 & 7.50 & 20 & 88.6 \\
ZPZLL-2 & 0.50 & 5.00 & 20 & 92.5 \\
ZPZLL-3 & 0.50 & 2.50 & 20 & 89.3
\end{tabular}


Table 2 Synthesis conditions of ZPLLs

\begin{tabular}{lllll}
\hline Sample & $\begin{array}{l}\text { ZPZLL } \\
(\mathrm{g})\end{array}$ & $\begin{array}{l}\text { TFA } \\
(\mathrm{mL})\end{array}$ & $\begin{array}{l}\text { HBr in acetic acid } \\
(33 \mathrm{wt} \%)(\mathrm{mL})\end{array}$ & Yield\% \\
\hline ZPLL-1 & 1.00 & 10 & 3.0 & 73.2 \\
ZPLL-2 & 1.00 & 10 & 3.0 & 68.7 \\
ZPLL-3 & 1.00 & 10 & 3.0 & 71.3
\end{tabular}

obtained was washed thoroughly with deionized water and dried under vacuum (Table 2).

\subsection{Preparation of the nanofibrous membranes}

Electrospinning solutions with a concentration of $20 \%(\mathrm{w} / \mathrm{v})$ were prepared by separately dissolving $1.00 \mathrm{~g}$ zein, ZPLL-1, ZPLL-2, and ZPLL-3 in 5.0 mL 1,1,1,3,3,3-hexafluoro-2propanol (HFIP). Electrospun nanofibrous membranes of zein, ZPLL-1, ZPLL-2, and ZPLL-3 were prepared by applying a voltage of $20 \mathrm{kV}$. The collector distance was controlled at $15 \mathrm{~cm}$ and the flow rate was $0.008 \mathrm{~mm} \mathrm{~min}^{-1}$.

The fiber morphologies of electrospun nanofibrous membranes were examined by SEM (JSM-6330F, Tokyo, Japan). The electrospun nanofibrous membranes were sputter-coated with gold and visualized by SEM at an accelerating voltage of $20 \mathrm{kV}$. Diameters of the nanofibers were measured using Image plus 6 . The average diameters were calculated from 300 random measurements.

The contact angle of the nanofibrous membranes was measured using KRUSS DSA10-MK. Each sample was measured three times to obtain the mean value.

\subsection{Degradation of nanofibrous membranes}

Zein, ZPLL-1, ZPLL-2, and ZPLL-3 nanofibrous membranes were cut to a size of $10 \times 10 \mathrm{~mm}^{2}$ and then immersed in PBS ( $\left.\mathrm{pH} 7.4\right)$ solution containing collagenase type I $\left(10 \mathrm{mg} \mathrm{mL}^{-1}\right)$. These membranes were removed after 7 days and washed three times with deionized water. After vacuum drying, the morphologies were observed by SEM.

\subsection{Extraction of neural stem cells (NSCs)}

A female pregnant SD rat was sacrificed 14 days after gestation. The NSCs from E14 rat embryos' cortex were cultured at $37{ }^{\circ} \mathrm{C}$ in a humid, $5 \% \mathrm{CO}_{2}$ incubator in a growth medium containing DMEM/F12 medium, 2\% StemPro neural supplement, $20 \mathrm{ng} \mathrm{mL}{ }^{-1}$ epidermal growth factor (EGF), $20 \mathrm{ng} \mathrm{mL}^{-1}$ basic fibroblastic growth factor (bFGF), 1\% GlutaMAX, and $1 \%$ penicillin-streptomycin solution. Cells were passaged every 3 days, digested with accutase, centrifuged, and resuspended at a density of $1 \times 10^{6}$ cells per $\mathrm{mL}$ in the precursor solution. After three day incubation, the NSCs suspensions were added in poly(lysine) coated 24-well plates and maintained another 4 hours at $37{ }^{\circ} \mathrm{C}$ in a humid $5 \% \mathrm{CO}_{2}$ incubator. Expression of nestin, a marker of NSCs, was assessed by immunocytochemistry.

\subsection{Seeding of NSCs on nanofibrous membranes}

Prior to cell seeding, zein, ZPLL-1, ZPLL-2, and ZPLL-3 nanofibrous membranes were cut into a $1 \mathrm{~cm}^{2}$ size and then sterilized by ultraviolet (UV) irradiation overnight for both top and bottom surfaces in a laminar flow hood. They were then placed in 24-well plate and NSCs were seeded on the electrospun membranes in each well at a density of $1 \times 10^{5}$ cells per $\mathrm{mL}$ in triplicate for culturing. Growth medium was added and cells were allowed to proliferate for up to 72 hours. Proliferation of the cells on each specimen was determined after different time intervals of 24,48 , and $72 \mathrm{~h}$, respectively. At each time point, the supernatant in each well was removed and dying reagents of LIVE/DEAD kits was added and the plate was then incubated for $45 \mathrm{~min}$ at $37^{\circ} \mathrm{C}$. The number of green cells was calculated under a random field of vision and at least 5 fields counted with each specimen for cell studies.

\subsection{Quantification of proliferation cells (CCK-8 assay)}

For cell proliferation studies, the cells were first allowed to attach to the specimens for 24 hours. Then cells were harvested from the membranes and seeded in 96-well culture plates at a density of $1 \times 10^{4}$ cells per well. Cells were incubated in the culture medium for $24 \mathrm{~h}$ after which it was replaced by $100 \mu \mathrm{L}$ of fresh medium in each well; $10 \mu \mathrm{L} \mathrm{CCK}-8$ solution was then added. After incubation for another $2 \mathrm{~h}$, the absorbance of each sample well was measured by a microplate reader at a wavelength of $450 \mathrm{~nm}$. Cells without incubation or using any materials and drugs were used as a control group and culture medium and CCK-8 solutions without cells were used as the blank control group. The viabilities were calculated and analysed following CCK-8 kit instructions.

\subsection{Cell differentiation on nanofibrous membranes}

Immunohistochemical analysis was performed to visualize differentiation of cells and the length of neurites. After 14 days of NSCs cultured in mixed differentiation media (2\% FBS), each well was washed with $1.5 \mathrm{~mL}$ of phosphate buffer saline (PBS) and fixed for $20 \mathrm{~min}$ with $1 \mathrm{~mL}$ of $4 \%$ formaldehyde. When necessary, the cells were permeabilized in $0.3 \%$ Triton X-100 for 15 min, followed by blocking with 2\% BSA in PBS for $30 \mathrm{~min}$ at room temperature. The cells were incubated with primary antibody overnight at $4{ }^{\circ} \mathrm{C}$. The following primary dilution antibodies were used to characterize three primary CNS lineages of neurons, oligodendrocytes, and astrocytes $\mathrm{v}$ in cultures: NeuN $(1: 300)$, O4 (1:100), and GFAP ( $1: 100)$. On the second day, each well was washed 3 times with PBS for 5 min. Appropriate secondary antibodies (1:500 dilution) were applied for $1 \mathrm{~h}$ at room temperature, then washed twice with PBS and with 4',6-diamidino-2-phenylindole dilactate (DAPI) for $5 \mathrm{~min}$ to stain nuclei, and then stored at $4{ }^{\circ} \mathrm{C}$ until fluorescent images were taken. Neurite length was measured as a linear distance between the tip of the neurite and the cell junction and collected only when neurite length was greater than $5 \mu \mathrm{m}$. Fluorescence images were captured using a color 
CCD camera (Optronics MagnaFire, Olympus) attached to a fluorescence microscope (IX-71, Olympus, Japan), and analyzed using Image $\mathrm{J}$ software.

The significance of the data was assessed using Student's $t$ test. Computer software (Sigmaplot 11.0, SPSS) was employed to calculate $p$ values, where $p<0.05$ was considered statistically significant.

\section{Results and discussion}

\subsection{Synthesis and characterization}

Fig. 1 shows the ${ }^{1} \mathrm{H}$ NMR spectra of zein, ZPZLL, and ZPLL in $\mathrm{d}_{6}$-DMSO. Since the structure of the polypeptide is similar to that of zein, most of the characteristic signals overlapped. The characteristic signals of zein such as $\mathrm{CH}_{3}$ groups at $0.8 \mathrm{ppm}$, $\mathrm{CH}_{2}$ groups at $1.2 \mathrm{ppm}$, and $\mathrm{NH}_{2}$ groups around $8.0 \mathrm{ppm}$ appeared in both ZPZLL and ZPLL spectra. ${ }^{33}$ The characteristic signal of methylene in a carbobenzoxy group appeared at 4.94 ppm which confirmed the successful grafting of PZLL on zein. On the other hand, the characteristic signal at $4.94 \mathrm{ppm}$, which disappeared in the spectrum of ZPLL, indicated completion of the deprotection reaction. Besides, the characteristic signal of methylene next to the secondary amine that shifted from $2.93 \mathrm{ppm}$ on the ZPZLL spectrum to $2.72 \mathrm{ppm}$ on the ZPLL spectrum also proves the deprotection reaction.

FTIR spectra of zein, ZPZLL, and ZPLL are shown in Fig. 2. Success of the reaction was confirmed by appearance of the bands at $743 \mathrm{~cm}^{-1}$ and $699 \mathrm{~cm}^{-1}$ in the spectrum of ZPZLL and the disappearance of these characteristic peaks, which belong to the out-of-plane bending vibration of a phenyl ring, in that of ZPLL. The secondary structure of zein could also be analyzed from its spectrum. The amide A band appearing between 2960 $\mathrm{cm}^{-1}$ and $3500 \mathrm{~cm}^{-1}$ belong to the stretching of the $\mathrm{N}-\mathrm{H}$ and $\mathrm{O}-\mathrm{H}$ bonds of the amino acids. ${ }^{33}$ The amide I bond appearing at $1660 \mathrm{~cm}^{-1}$ belongs to stretching of the carbonyl $(\mathrm{C}=\mathrm{O})$ of amide groups of the peptide. The amide II band at $1549 \mathrm{~cm}^{-1}$

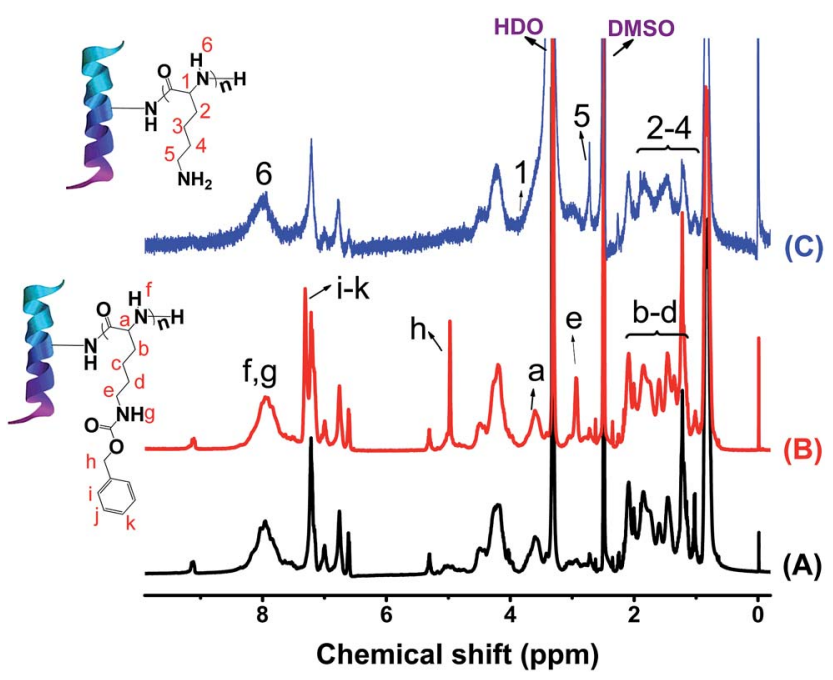

Fig. $1{ }^{1} \mathrm{H}$ NMR spectra of (A) zein, (B) ZPZLL, and (C) ZPLL in $d_{6}-D M S O$.

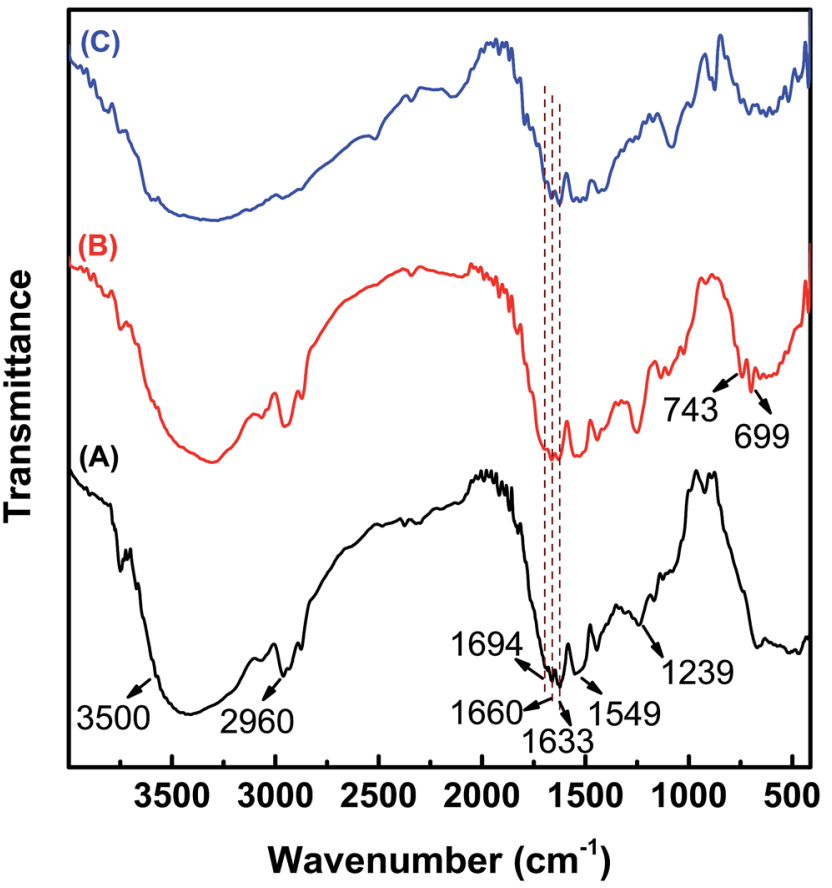

Fig. 2 FTIR spectra of (A) zein, (B) ZPZLL, and (C) ZPLL.

corresponds to angular deformation vibrations of the $\mathrm{N}-\mathrm{H}$ bond and $\mathrm{C}-\mathrm{H}$ stretching vibrations. Moreover, the amide III band at $1239 \mathrm{~cm}^{-1}$ belongs to axial deformation vibrations of $\mathrm{C}-\mathrm{N}$ bond and $\mathrm{C}=\mathrm{O}$ bending vibrations. ${ }^{36}$ Also, the absorption band at $1660 \mathrm{~cm}^{-1}$ indicates the existence of $\alpha$-helices. Signals of $\beta$ sheet appear at $1633 \mathrm{~cm}^{-1}$ and $1694 \mathrm{~cm}^{-1} \cdot{ }^{36,37}$ The characteristic peaks of zein appeared in both ZPZLL and ZPLL spectra, thus proving the structure of zein had not been destroyed after modification.

The content of PLL in ZPLL-1, ZPLL-2, and ZPLL-3 were calculated from elemental analysis, which are $1.46 \%, 3.57 \%$, and $6.18 \%$, respectively (PLL content $=$ the mass of PLL/the mass of zein $\times 100 \%$ ).

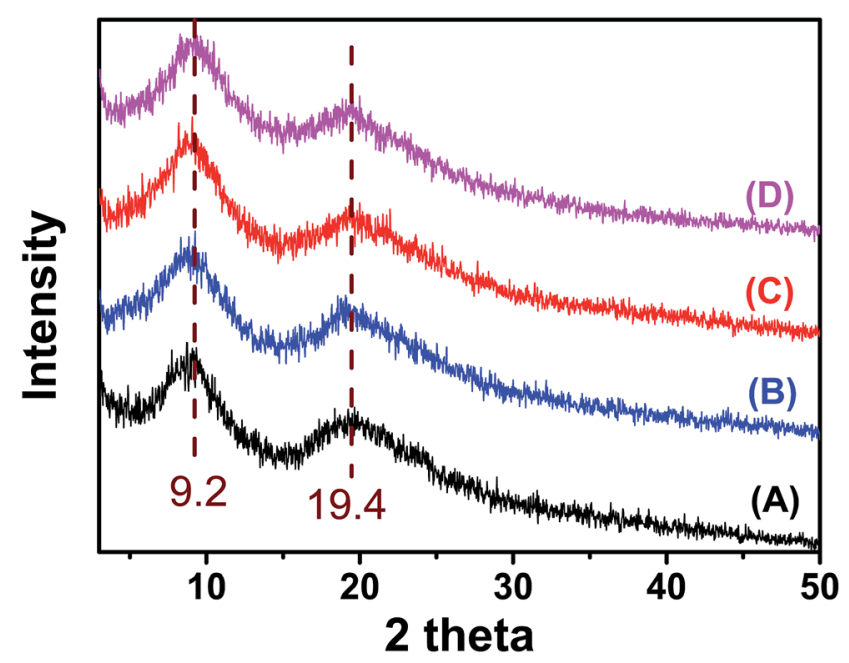

Fig. 3 XRD spectra of (A) zein, (B) ZPLL-1, (C) ZPLL-2, and (D) ZPLL-3. 
X-ray diffraction spectra of zein, ZPLL-1, ZPLL-2, and ZPLL-3 are shown in Fig. 3. Two peaks around $9.2^{\circ}$ and $19.4^{\circ}$ appeared in all zein, ZPLL-1, ZPLL-2, and ZPLL-3 spectra; these are related to $\alpha$-helix and $\beta$-sheet structures of protein. ${ }^{36}$ The intensity of these two peaks did not show any change after being modified by poly(L-lysine), showing that the $\alpha$-helix and $\beta$-sheet structures of zein were not influenced.
Since the solubility of protein in water is greatly affected by the $\alpha$-helix and $\beta$-sheet structures, the conformation of zein is critical for the stability of electrospun membranes in water. ${ }^{38}$ Based on this consideration, the conformation of zein was investigated by XRD to make sure the membranes could be stably used as a cell scaffold.
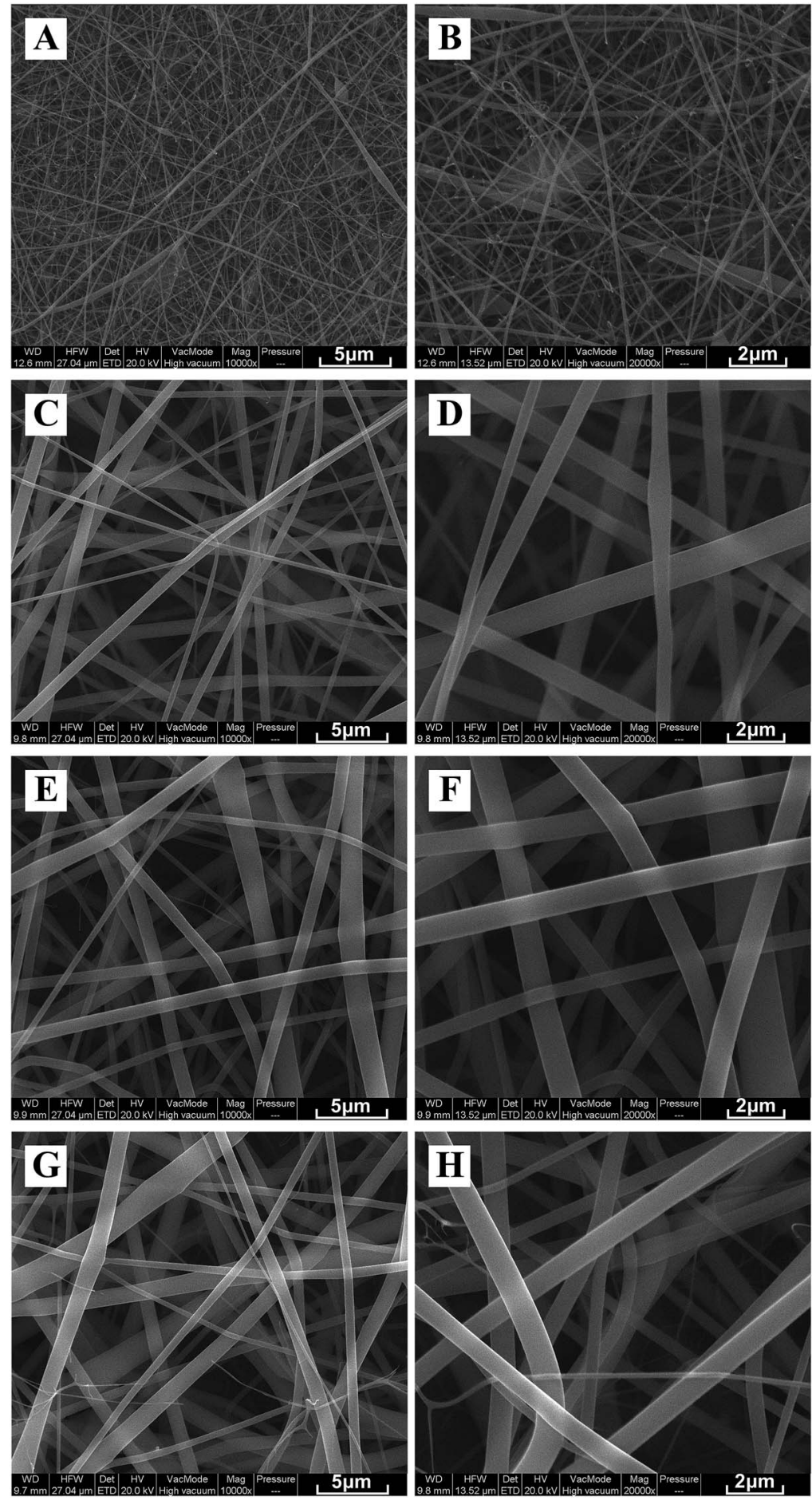
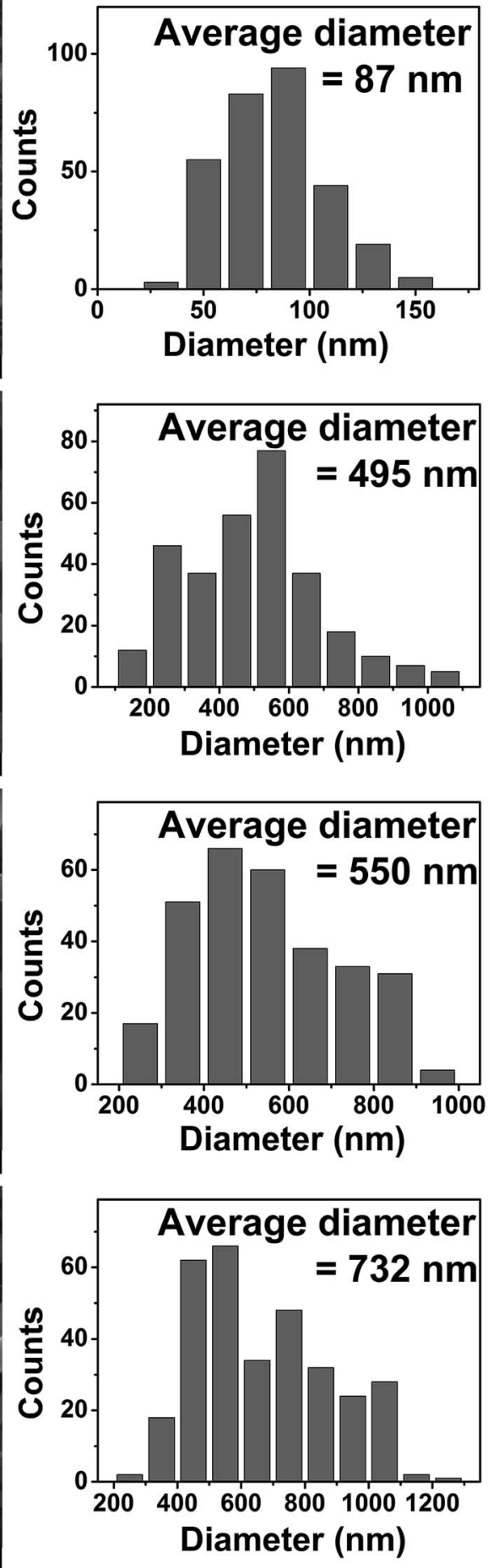

Fig. 4 SEM pictures of electrospun (A, B) zein membranes, (C, D) ZPLL-1 membranes, (E, F) ZPLL-2 membranes, and (G, H) ZPLL-3 membranes under a magnification of $10000 \times$ and $20000 \times$ respectively. 


\subsection{Characterization of the nanofibrous membranes}

SEM pictures of electrospun zein and ZPLL nanofibers are shown in Fig. 4. The average diameter of pure zein nanofibers was $87 \mathrm{~nm}$. ZPLL-1, ZPLL-2, and ZPLL-3 nanofibers had larger diameters with average diameters of $495 \mathrm{~nm}, 550 \mathrm{~nm}$, and $732 \mathrm{~nm}$, respectively; this indicated that the diameters of ZPLL nanofibers became larger as the content of poly(L-lysine) increased. The molecular weight of ZPLL also increased with the content of PLL. This could lead to an increase of the viscosity of the electrospinning solutions and then cause an increasing of fiber diameter.

\subsection{Characterization of the contact angle of the nanofibrous membranes}

Water contact angle pictures of electrospun zein and ZPLL membranes are shown in Fig. 5. The contact angle of these membranes decreased from $129.8 \pm 2.3^{\circ}$ with pure zein to $123.5 \pm 1.6^{\circ}$ with ZPLL-1, $118.4 \pm 1.5^{\circ}$ with ZPLL-2, and 110.9 $\pm 2.1^{\circ}$ with ZPLL-3 with increasing content of poly(L-lysine). It has been reported that poor hydrophilicity can lead to a reduction in the ability of cell adhesion, migration, proliferation, and differentiation. ${ }^{39}$ Because of the hydrophobic property of zein, hydrophilic modification is needed. Contact angle results show the hydrophilic property could be improved by modifying poly(L-lysine), which might be beneficial for the adhesion, proliferation, and differentiation of neural stem cells.

\subsection{Degradation of the nanofibrous membranes}

The morphological changes in zein, ZPLL-1, ZPLL-2, and ZPLL-3 membranes after 7 days of in vitro enzymatic degradation are shown in Fig. 6 . The holes and the broken area on the nanofibers indicated degradation of the membranes which proved the biodegradability of zein materials. This character in materials is important for biomaterials used in vivo; as an organism regenerates, the implant material could gradually disappear without the need of being removed by an operation.

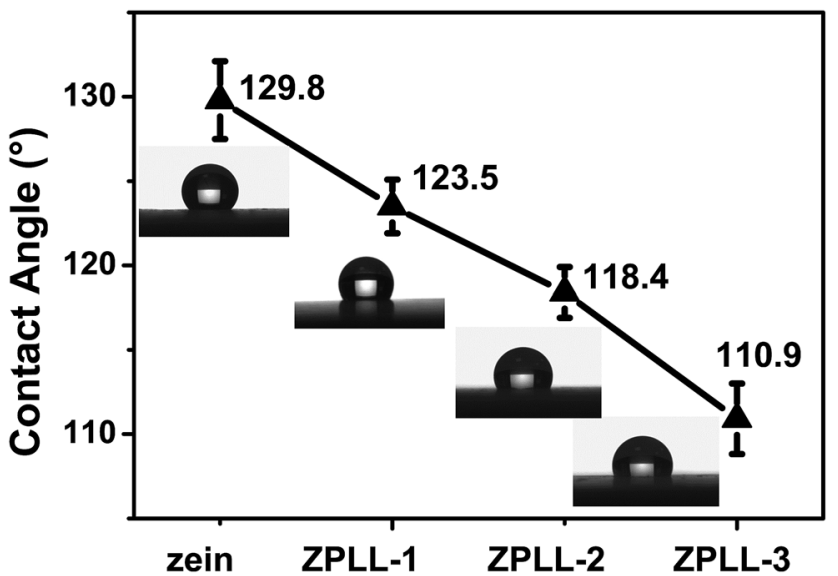

Fig. 5 Water contact angle pictures of electrospun zein, ZPLL-1, ZPLL-2, and ZPLL-3 membranes.
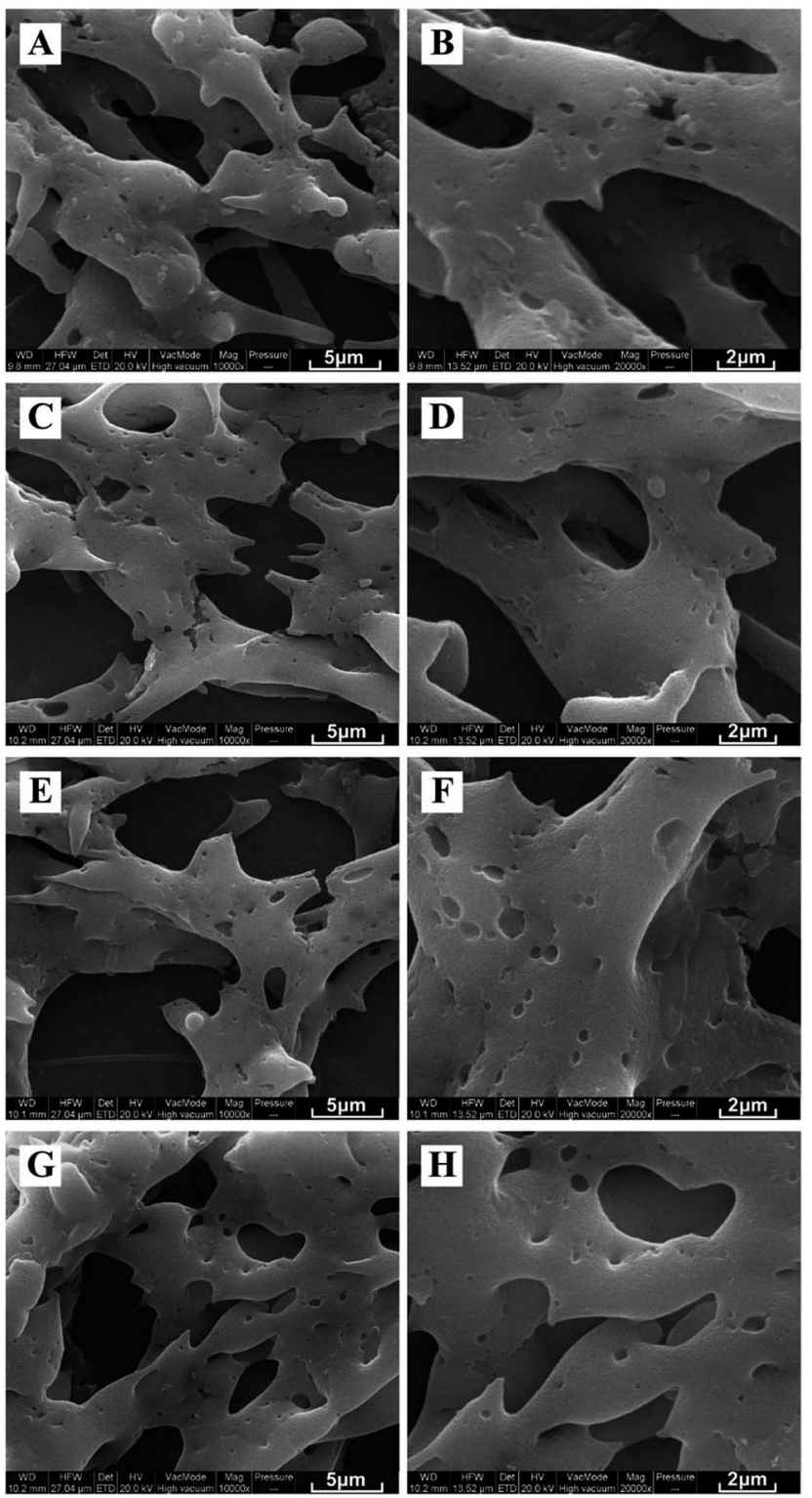

Fig. 6 Morphological changes in electrospun (A, B) zein membranes, (C, D) ZPLL-1 membranes, (E, F) ZPLL-2 membranes, and $(G, H)$ ZPLL-3 membranes after 7 days of in vitro enzymatic degradation under magnification of $10000 \times$ and $20000 \times$, respectively.

\subsection{Morphology of proliferated NSCs on membranes and quantification of proliferation cells (CCK-8 assay)}

NSCs were isolated from the whole cerebral cortex of E14 SD rats because formation of neurospheres is considered the gold standard for identification of NSCs. The NSCs we cultured aggregated as free-floating neurospheres (Fig. S1A $\dagger$ ), and were observed under a phase contrast microscope after 3 days of culture. Then, the NSCs were identified using nestin, a known protein marker of NSCs, and they showed nestin positive immunoreactivity (Fig. S1B $\dagger$ ). These data indicated that the NSCs maintained an undifferentiated status in their growth medium. 
(A)
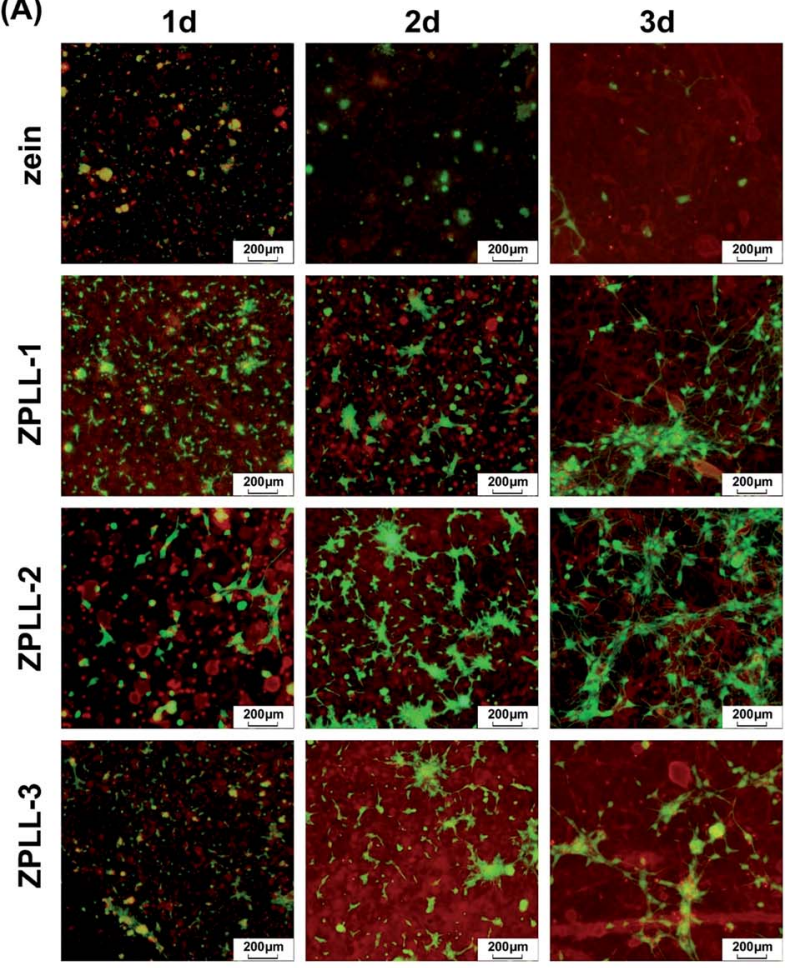

(B)

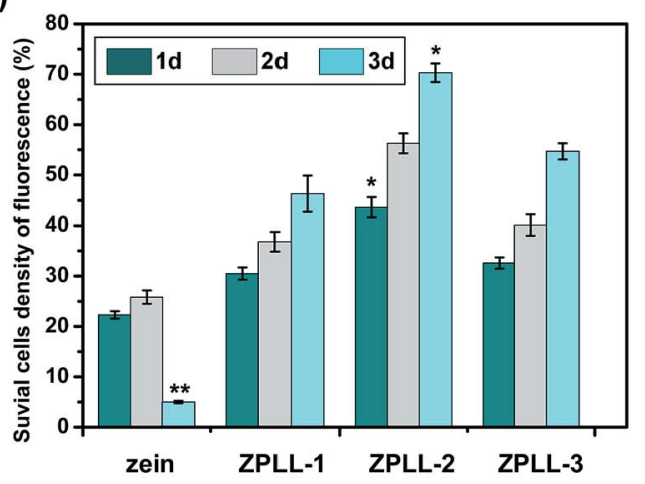

(C)

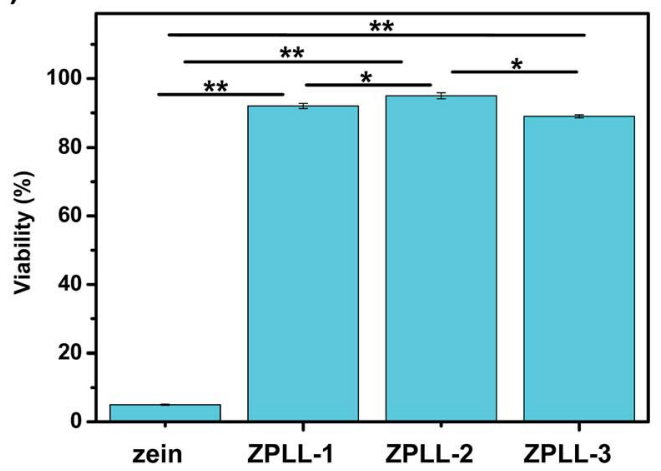

Fig. 7 (A) Fluorescent photographs of NSCs and (B) live cells density on zein, ZPLL-1, ZPLL-2, and ZPLL-3 electrospun membranes after culturing for 1,2 , and 3 days. (C) Cell viabilities of NSCs on zein, ZPLL-1, ZPLL-2, and ZPLL-3 electrospun membranes determined by CCK- 8 $(* P<0.05, * * P<0.01)$.
Fig. 7A shows representative images of NSCs that had been adhered to the surfaces of the zein nanofibrous for $24 \mathrm{~h}$, day 2 , and day 3. Green cells stained by calcein-AM were observed under $485 \pm 10 \mathrm{~nm}$ and red cells stained by ethidium homodimer (EthD-I) were observed under $530 \pm 12.5 \mathrm{~nm}$. It is noticeable that the nanofibers of these membranes were also stained by EthD-I showing red fluorescence, which made it easy to observe them on the membranes. NSCs survival and neuron synapses were observed which indicated signs of differentiation in the four groups. Overall survival of the grafted NSCs was quantified for each group.

The average live cells density on zein, ZPLL-1, ZPLL-2, and ZPLL-3 electrospun membranes were determined to be $25.8 \pm$ $1.1 \%, 36.8 \pm 2.1 \%, 56.3 \pm 2.3 \%$, and $40.1 \pm 1.6 \%$, respectively (Fig. 7B). The percentage of live cells was highest on ZPLL-2 membranes. Conversely, the percentage of live cells was lowest on zein membranes. No significant difference in viability was found between ZPLL-1 and ZPLL-3 membranes.

Further increasing the culturing time to days 2 and 3, the cultured cells were observed to be well-spread and formed intercellular tight junctions with adjacent cells on the last three membrane's surfaces. The live cells density growth by differentiation on ZPLL-1 and ZPLL-3 membrane surfaces was slightly lower than the corresponding one on ZPLL-2 membrane surfaces. On day 3 , almost no green cells were observed on pure zein membranes.

To determine viability of NSCs on the membranes, cells were analyzed by CCK-8 assay as described and detailed in the Materials and methods section. As seen in Fig. 7C, NSCs viability was $92 \pm 0.76 \%$ on ZPLL-1 membranes, and it increased to $95 \pm 0.87 \%$ on ZPLL-2 membranes, while reaching $89 \pm 0.43 \%$ on ZPLL-3 membranes; the viability on pure zein electrospun membranes decreased sharply, only measuring $5 \pm$ $0.56 \%$. It may be concluded that the membranes were more suitable for cell culturing after modification by poly(L-lysine); ZPLL-2 membranes show the highest viability which declare the best biocompatibility.

\subsection{Differentiation of NSCs on membranes}

To evaluate the differentiation of seeded NSCs on nanofibrous membranes, we stained the cells with NeuN O4 $(1: 100)$ and GFAP $(1: 100)$. NSCs were differentiated into all three primary CNS lineages of neurons, astrocytes, and oligodendrocytes on the four groups in mixed differentiation media (2\% FBS). As seen in Fig. 8A, the total percentage of NSCs differentiation toward neurons increased dramatically from $34 \pm 3.8 \%$ on ZPLL-1 membranes to $52 \pm 6.3 \%$ on ZPLL- 2 membranes and significantly decreased to $41 \pm 3.3 \%$ on ZPLL-3 membranes. Conversely, the percentage of NSCs differentiation to astrocytes on ZPLL-1, ZPLL-3, and ZPLL-3 membranes were determined to be $72 \pm 4.6 \%, 46 \pm 1.3 \%$, and $57 \pm 2.1 \%$, respectively. Oligodendrocyte differentiation was not well supported by all the groups under the same differentiation conditions. Almost no O4-positive cells were found on these nanofibrous membranes. Neurite length was calculated for neurons differentiated by NSCs cultured on three different samples (Fig. 8B). In addition, 

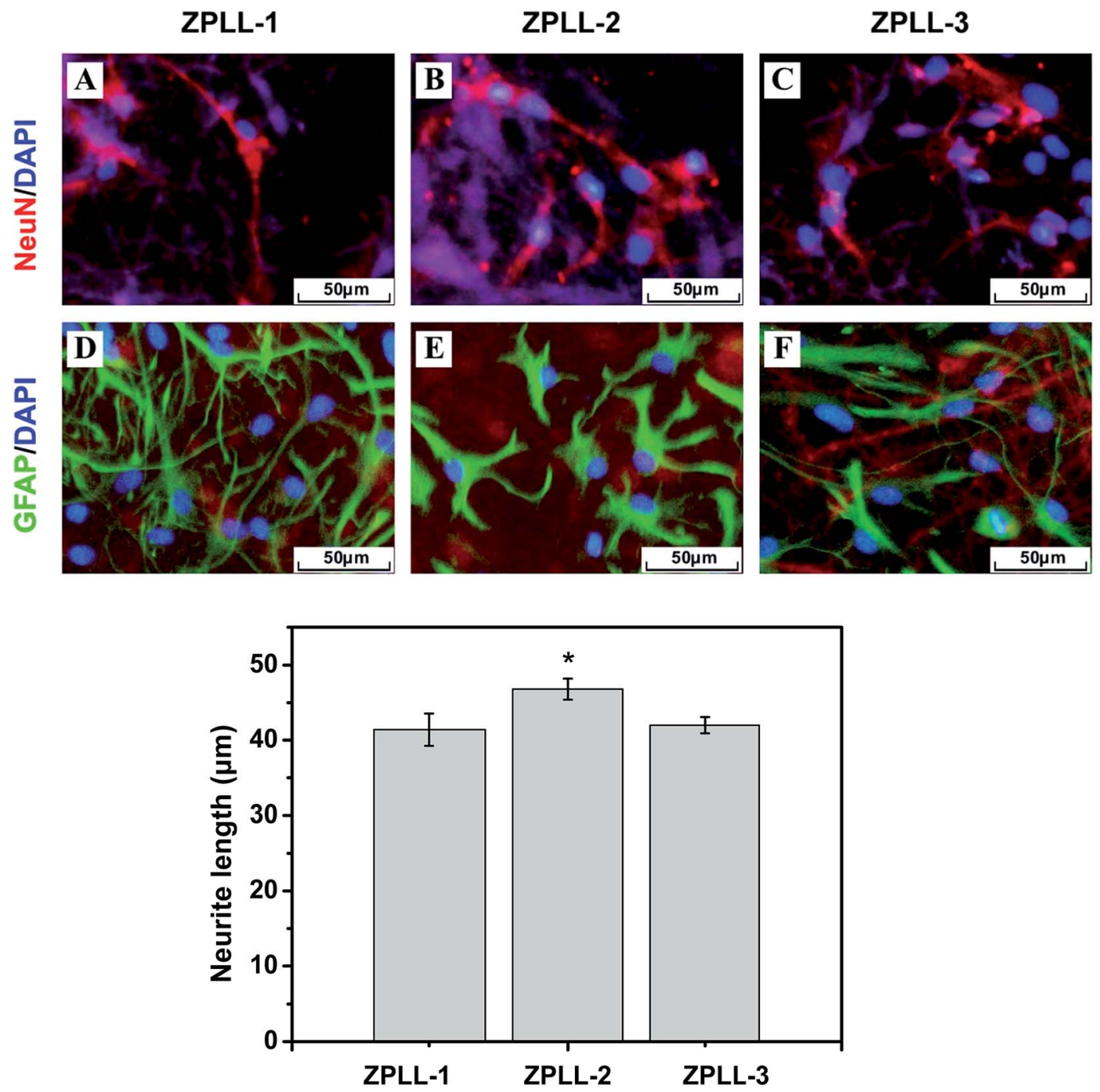

Fig. 8 Differentiation of NSCs after culturing for 14 days in vitro. (A-F) Cells cultured on ZPLL-1, ZPLL-2, and ZPLL-3 membranes were immunostained with NeuN for (A-C) neurons and (D-F) GFPA for astrocytes (*P<0.05; $n=5$ ).

the maximum length of neurites projecting from the neurons cultured on ZPLL-2 samples was significantly longer than that of neurites projecting from the neurons developed on ZPLL-1 and ZPLL-3 membranes. ZPLL-2 membranes, therefore, must be capable of enhancing the length of neurite extension.

\section{Conclusions}

Zein is a promising material in biomedical fields as a natural protein from corn. However, its application is limited by its hydrophobic property. In this work, we modified zein with poly(L-lysine), which has a similar structure to protein, using a chemical modification method. Since poly(L-lysine) is watersoluble, the hydrophilicity of zein was able be improved. Also, the hydrophobic and hydrophilic performances of zein could be easily controlled by the content amount of poly(L-lysine). Poly(Llysine) modified zein membranes also show excellent biocompatibility and biodegradability which makes them a promising material for biomaterial research areas. In addition to promoting cell adhesion and proliferation, differentiation was also influenced by the content amounts of poly(L-lysine). These results indicate that the poly(L-lysine) modified zein nanomembranes are promising biomaterials for nerve repair and regeneration.

\section{Acknowledgements}

This work was supported by the Special Funds for Public Welfare Research and Capacity Building of Guangdong Province in China (2014A010105025), the Key Project of Guangdong Provincial Natural Science Fund in China (S2013020012818) and Guangdong Provincial Natural Science Foundation of China (2015A030313186).

\section{References}

1 E. M. Jeffries and Y. Wang, Biotechnol. Bioeng., 2012, 6, 15711582. 
2 X. Navarro, M. Vivó and A. Valero-Cabré, Prog. Neurobiol., 2007, 82, 163-201.

3 W. W. Campbell, Clin. Neurophysiol., 2008, 119, 1951-1965. 4 L. B. Dahlin, Scand. J. Surg., 2008, 310-316.

5 E. L. Whitlock, S. H. Tuffaha, J. P. Luciano, Y. Yan, D. A. Hunter, C. K. Magill, A. M. Moore, A. Y. Tong, S. E. Mackinnon and G. H. Borschel, Muscle Nerve, 2009, 39, 787-799.

6 U. Assmann, A. Szentivanyi, Y. Stark, T. Scheper, S. Berski, G. Dräger and R. H. Schuster, J. Mater. Sci.: Mater. Med., 2010, 21, 2115-2124.

7 M. Siemionow, M. Bozkurt and F. Zor, Microsurgery, 2010, 30, 574-588.

8 M. Georgiou, S. C. J. Bunting, H. A. Davies, A. J. Loughlin, J. P. Golding and J. B. Phillips, Biomaterials, 2013, 34, 7335-7343.

9 N. Mokarram, A. Merchant, V. Mukhatyar, G. Patel and R. V. Bellamkonda, Biomaterials, 2012, 33, 8793-8801.

10 G. C. de Ruiter, M. J. Malessy, M. J. Yaszemski, A. J. Windebank and R. J. Spinner, Neurosurg Focus, 2009, 26, 1-9.

11 T. Murakami, Y. Fujimoto, Y. Yasunaga, O. Ishida, N. Tanaka, Y. Ikuta and M. Ochi, Brain Res., 2003, 974, 17-24.

12 L. Ghasemi-Mobarakeh, M. P. Prabhakaran, M. Morshed, M. H. Nasr-Esfahani and S. Ramakrishna, Mater. Sci. Eng. C., 2010, 30, 1129-1136.

13 A. J. Reid, A. C. de Luca, A. Faroni, S. Downes, M. Sun, G. Terenghi and P. J. Kingham, Neurosci. Lett., 2013, 544, 125-130.

14 L. Cai, J. Lu, V. Sheen and S. Wang, Biomacromolecules, 2012, 13, 342-349.

15 J. Zhang, K. Qiu, B. Sun, J. Fang, K. Zhang, H. EI-Hamshary, S. S. Al-Deyab and X. Mo, J. Mater. Chem. B, 2014, 2, 79457954.

16 S. Kakinoki and T. Yamaoka, J. Mater. Chem. B, 2014, 2, 5061. 17 T. R. Kuang, F. Chen, L. Q. Chang, Y. N. Zhao, D. J. Fu, X. Gong and X. F. Peng, Chem. Eng. J., 2017, 1017-1025.

18 V. Chiono, S. Sartori, A. Rechichi, C. Tonda-Turo, G. Vozzi, F. Vozzi, M. D'Acunto, C. Salvadori, F. Dini, G. Barsotti, F. Carlucci, S. Burchielli, S. Nicolino, C. Audisio, I. Perroteau, P. Giusti and C. Gianluca, Macromol. Biosci., 2011, 2, 245-256.
19 A. Cooper, N. Bhattarai and M. Zhang, Carbohydr. Polym., 2011, 85, 149-156.

20 Y. Yang, X. Gu, R. Tan, W. Hu, X. Wang, P. Zhang and T. Zhang, Biotechnol. Lett., 2004, 26, 1793-1797.

21 B. Liu, C. Yao, S. Hsu, T. Yeh, Y. Chen and S. Kao, J. Biomater. Appl., 2004, 19, 21-34.

22 J. Chang, T. Ho, H. Lee, Y. Lai, M. Lu, C. Yao and Y. Chen, Artif. Organs, 2009, 33, 1075-1085.

23 Y. Yang, F. Ding, J. Wu, W. Hu, W. Liu, J. Liu and X. Gu, Biomaterials, 2007, 28, 5526-5535.

24 S. Yoshii and M. Oka, Brain Res., 2001, 888, 158-162.

25 F. Stang, H. Fansa, G. Wolf, M. Reppin and G. Keilhoff, Biomaterials, 2005, 26, 3083-3091.

26 M. M. Laura and E. S. Shelly, Curr. Opin. Biotechnol., 2013, 887-892.

27 L. Cai, J. Lu, V. Sheen and S. Wang, Biomacromolecules, 2012, 13, 1663-1674.

28 E. Corradini, P. Curti, A. Meniqueti, A. Martins, A. Rubira and E. Muniz, Int. J. Mol. Sci., 2014, 15, 22438-22470.

29 R. Shahlori, G. I. N. Waterhouse, A. R. J. Nelson and D. J. McGillivray, J. Mater. Chem. B, 2015, 3, 6213-6223.

30 C. Liu, Y. Chen, X. Wang, J. Huang, P. R. Chang and D. P. Anderson, J. Mater. Sci., 2010, 45, 6775-6785.

31 C. Yao, X. Li and T. Song, J. Biomater. Sci., Polym. Ed., 2007, 18, 731-742.

32 S. Torres-Giner, M. J. Ocio and J. M. Lagaron, Carbohydr. Polym., 2009, 77, 261-266.

33 K. Shi, Y. Huang, H. Yu, T. Lee and Q. Huang, J. Agric. Food Chem., 2011, 59, 56-61.

34 J. Wang, Z. Ye, S. Zheng, L. Chen, Y. Wan, Y. Deng and R. Yang, Brain Res., 2016, 1634, 34-44.

35 N. Chen, J. Cen, J. Wang, G. Qin, L. Long, L. Wang, F. Wei, Q. Xiang, D. Y. B. Deng and Y. Wan, Crit. Care Med., 2016, 44, 146-157.

36 Y. Han, Q. Xu, Z. Lu and J. Wang, Colloids Surf., B, 2014, 120, 55-62.

37 D. M. Byler and H. Susi, Biopolymers, 1986, 469-487.

38 S. Das, D. Pati, N. Tiwari, A. Nisal and S. Sen Gupta, Biomacromolecules, 2012, 13, 3695-3702.

39 C. H. Kim, M. S. Khil, H. Y. Kim, H. U. Lee and K. Y. Jahng, J. Biomed. Mater. Res., Part B, 2005, 283-290. 